\title{
Trasplante de Progenitores Hematopoyéticos en Pediatría: 10 Años de Experiencia
}

\author{
GUSTAVO DUFORT Y ÁLVAREZ ${ }^{1}$, MARIELA CASTIGLIONI ${ }^{1}$, CAROLINA PAGÉS $^{1}$, \\ AGUSTÍN DABEZIES ${ }^{1}$, JORGE DECARO ${ }^{2}$, LUIS CASTILLO ${ }^{3}$ \\ 1. Staff de la Unidad de Trasplante de Médula Ósea Pediátrica. Asociación Española. \\ 2. Director del Departamento de Medicina Transfusional. Asociación Española. \\ 3. Director de la Unidad de Trasplante de Médula Ósea Pediátrica. Asociación Española. \\ Unidad de Trasplante de Médula Ósea Pediátrica. Asociación Española Primera de Socorros Mutuos.
}

\begin{abstract}
\section{Hematopoietic Stem Cell Transplantation in Pediatrics: 10 years experience}

Background: In Uruguay the survival estimates for pediatric patients with cancer are comparable to those in developed countries. With the aim of improving outcomes in patients with worse prognosis and provide therapeutic options for treating some non malignant diseases, we started a hematopoietic stem cell transplantation (HSCT) program in 1997, with the financial support of the National Resources Fund (Fondo Nacional de Recursos-FNR). Patients and Methods: The unit was created at the Asociación Española Hospital and counts with three rooms with a sophisticated isolation system. Isolation measures and guidelines for preventing opportunistic infections were strictly enforced. HSCT indications followed the national FNR guidelines according to recommendations of international groups. International standards were used for the patients management. Results: In 10 years 131 transplants were performed in 129 patients. The 10 year overall survival estimate was $43.9 \pm 4.6 \%$. Transplant related mortality was $9.3 \%$ and the main cause of death was infections. In allogenic HSCT acute graft versus host disease was the leading cause of death. There were three patients who had hither to secondary malignancies. Conclusions: The results, as well as past experience in these 10 years are encouraging. The utility of the procedure in many of the pathologies was analyzed and demonstrated. In the future our goal is to offer this treatment to all patients in which the HSCT is indicated, including those with no compatible family donor.
\end{abstract}

(Key words: Hematopoietic stem cell, transplantation, bone marrow transplantation, leukemia, myeloid, acute, precursor cell lymphoblastic leukemia-lymphoma, lymphoma, neoplasms, neuroblastoma).

Archivos de Pediatría del Uruguay 2008; 79 (3)

\section{RESUMEN}

Introducción: En Uruguay la sobrevida de los niños con cáncer es comparable a la de los países desarrollados. Con el objetivo de mejorar los resultados en pacientes de peor pronóstico y ofrecer opciones terapéuticas curativas a algunas enfermedades no malignas, en el año 1997 se inició un programa de trasplante de progenitores hematopoyéticos (TPH) en pediatría con el financiamiento del Fondo Nacional de Recursos (FNR). Pacientes

Correspondencia a:

Gustavo Dufort y Álvarez

E-mail: gdufort@chasque.net 
y Métodos: La unidad de trasplante fue creada en el sanatorio de la Asociación Española compuesta por tres habitaciones dotadas de sistemas sofisticados de aislamiento. Se implantaron medidas estrictas de aislamiento invertido y medidas de prevención de infecciones oportunistas protocolizadas. Las indicaciones de los trasplantes estuvieron reguladas por las normativas del FNR de acuerdo con recomendaciones internacionales. El manejo de los pacientes estuvo de acuerdo a los estándares internacionales. Resultados: En 10 años se realizaron 131 TPH en 129 pacientes, 39 alogénicos y 92 autólogos. La sobrevida global estimada a los 10 años fue $43,9 \pm 4,6 \%$. La muerte relacionada con el trasplante fue de 9,3\%, y la principal causa fueron las infecciones. La enfermedad injerto contra huésped aguda fue la principal causa de muerte en los TPH alogénicos. Hubo tres pacientes que presentaron hasta el momento segundas neoplasias. Conclusiones: Los resultados obtenidos, así como la experiencia acumulada en estos 10 años son alentadores y demuestran la utilidad del procedimiento en muchas de las patologías analizadas. En el futuro nuestro objetivo será poder ofrecerles este tratamiento a todos los pacientes en el cual el TPH esté indicado, incluidos aquellos sin donantes familiares compatibles. (Palabras clave: Trasplante de células madre, hematopoyéticas, trasplante de médula ósea, leucemia mieloide aguda, leucemia- linfoma linfoblástico de células precursoras, linfoma, neoplasias, neuroblastoma). Archivos de Pediatría del Uruguay 2008; 79 (3)

ESTE TRABAJO LO PUEDE ENCONTRAR EN EXTENSO EN WWW.SciELO.ORG 